\title{
On limits of dense packing of equal spheres in a cube
}

\author{
Milos Tatarevic \\ Alameda, CA 94501, U.S.A. \\ milos.tatarevic@gmail.com
}

Submitted: Oct 8, 2013; Accepted: Jan 27, 2015; Published: Feb 16, 2015

Mathematics Subject Classifications: 52C17, 05B40

\begin{abstract}
We examine packing of $n$ congruent spheres in a cube when $n$ is close but less than the number of spheres in a regular cubic close-packed (сср) arrangement of $\left\lceil p^{3} / 2\right\rceil$ spheres. For this family of packings, the previous best-known arrangements were usually derived from a ccp by omission of a certain number of spheres without changing the initial structure. In this paper, we show that better arrangements exist for all $n \leqslant\left\lceil p^{3} / 2\right\rceil-2$. We introduce an optimization method to reveal improvements of these packings, and present many new improvements for $n \leqslant 4629$.
\end{abstract}

\section{Introduction}

We consider the problem of finding the densest packings of congruent, non-overlapping, spheres in a cube. Equivalently, we can search for an arrangement of points inside a unit cube so that the minimum distance between any two points is as large as possible. The maximum separation distance of $n$ points in $[0,1]^{3}$ we denote by $d_{n}$.

To our knowledge, the optimality of $d_{n}$ is proved for $n=2,3,4,5,6,8,9$ [10], $n=10$ [11] and $n=14$ [6]. Optimality of $d_{n}$ was conjectured for an infinite family of packings where $\left\lceil p^{3} / 2\right\rceil$ spheres are arranged in a cubic close-packed (ccp) structure [3]. We denote by $g(p)=\left\lceil p^{3} / 2\right\rceil$ the number of spheres in these packings, with a maximum separation distance denoted by $d_{p}^{\prime}=\sqrt{2} /(p-1)$.

In this paper, we examine arrangements when $n$ is close, but less than $g(p)$. For this family of packings $d_{n}=d_{p}^{\prime}$ is often assumed, to mean that the densest known arrangements are derived from ccp by omission of a certain number of spheres without changing the initial structure. Limiting values though were not provided. It was conjectured that $d_{n}$ is constant in the range $12 \leqslant n \leqslant 14$ [3] and $29 \leqslant n \leqslant 32$ [2]. A better packing was found for $n=12$ [2]. Similarly, previous search results showed the same trend for $60 \leqslant n \leqslant 63$, $103 \leqslant n \leqslant 108, \ldots, 817 \leqslant n \leqslant 864[5,7,12]$.

In Section 2 we show that most of the listed packings can be improved by proving that $d_{n}>d_{p}^{\prime}$ for all $n \leqslant g(p)-2$. We also provide a lower bound for these improvements. 
In Section 3, we introduce an optimization method and improve the lower bound for $4 \leqslant p \leqslant 21$. We show that the described procedure can be used as a good packing method when $n$ is slightly smaller than $g(p)$. We run search to determine improvements for other packings when $n=g(p)-r$, for $3 \leqslant r \leqslant 6, r<p$ and $4 \leqslant p \leqslant 12$.

\section{Existence of improved packings}

To simplify our notation, we will assume that the radius of all spheres in the packing is 1 and that our task is to determine the smallest size of a cube that contains all spheres.

We denote the family of all finite sets of points such that the distance between any two points is at least 2 by

$$
\mathcal{F}=\left\{S \subset \mathbb{R}^{3}:\left\|s_{1}-s_{2}\right\| \geqslant 2 \text { for all distinct } s_{1}, s_{2} \in S\right\} .
$$

Let $S_{n}=\left\{s_{1}, \ldots, s_{n}\right\} \in \mathcal{F}$ and let $D_{c}\left(s_{i}, s_{j}\right)$ be the Chebyshev distance between any two points $s_{i}, s_{j} \in S_{n}$. The smallest edge length of a cube, with edges parallel to the axes, such that it contains all points $S_{n}$ is equal to

$$
D\left(S_{n}\right)=\max \left\{D_{c}\left(s_{i}, s_{j}\right): 1 \leqslant i<j \leqslant n\right\} .
$$

We notice that the maximum separation distance $d_{n}$ can also be given as

$$
d_{n}=\max \frac{2}{D\left(S_{n}\right)} .
$$

Theorem. The maximum separation distance of $n$ points contained in a closed region bounded by a unit cube is larger than $\sqrt{2} /(p-1)$ for all $n \leqslant\left\lceil p^{3} / 2\right\rceil-2$.

Proof. Let $C_{p}$ be a closed region bounded by a cube with an edge length $D_{p}=2 / d_{p}^{\prime}=$ $(p-1) \sqrt{2}$, where $C_{p}$ is defined by $C_{p}=\left[0, D_{p}\right]^{3}$. Let $G_{p}$ be a set of $g(p)$ sphere centers in a ccp arrangement, such that $G_{p} \in \mathcal{F}, G_{p} \subset C_{p}$ and $\langle 0,0,0\rangle \in G_{p}$ (see Figure 1).

For two sets of points $A, B \in \mathcal{F}$ let

$$
h(A, B)=\min \{\|a-b\|-2: a \in A, b \in B\}
$$

and let $L_{p}=G_{p} \backslash G_{p-1}$ (see Figure 2(a) for an example). We denote the improved packing of $g(p)-2$ points by $P_{p}=\left\{s_{1}, \ldots, s_{g(p)-2}\right\} \in \mathcal{F}$ such that

$$
\begin{gathered}
P_{p} \subset C_{p}, \\
h\left(P_{p}, L_{p+1}\right)>0 .
\end{gathered}
$$

Equations (1) and (2) directly imply that if these statements are true, then $D\left(P_{p}\right)<D_{p}$.

Let $T_{p}=\tau\left(L_{p}\right)$ be a set of points created by the translation of points from $L_{p}$ by the function $\tau$ such that

$$
T_{p} \in \mathcal{F}, T_{p} \subset C_{p}, h\left(P_{p-1}, T_{p}\right) \geqslant 0,
$$




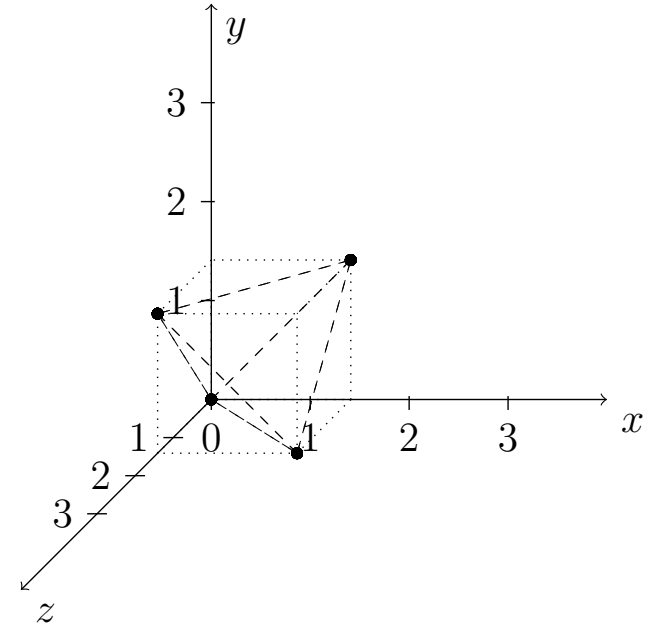

(a) $G_{2}$

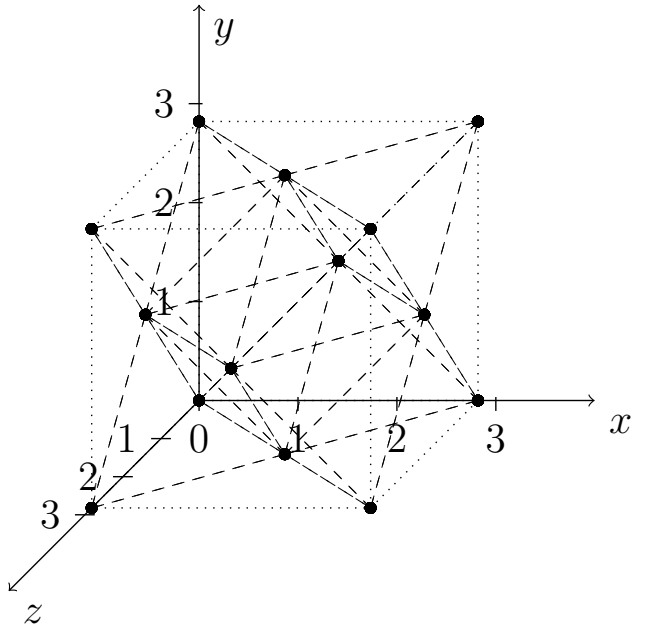

(b) $G_{3}$

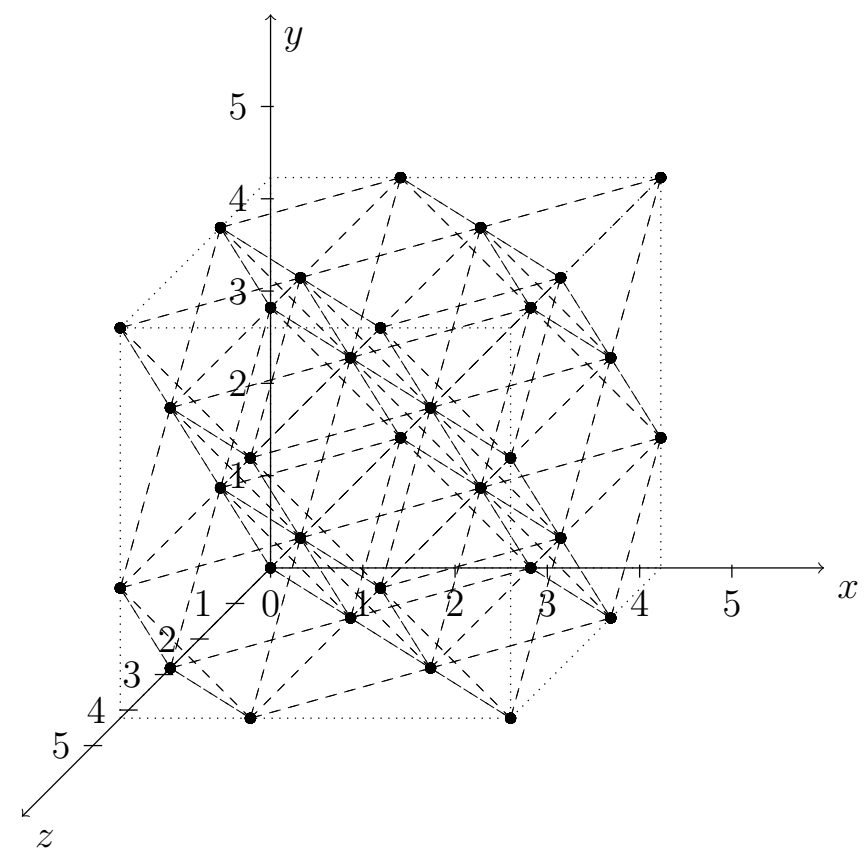

(c) $G_{4}$

Figure 1: An illustration of arrangements $G_{2}, G_{3}$ and $G_{4}$.

$$
h\left(T_{p}, L_{p+1}\right)>0
$$

for all $p>2$. If such a set $T_{p}$ exists, then we can state that

$$
P_{p}=P_{p-1} \cup T_{p}, \text { for all } p>2 .
$$

We can easily show that $P_{2}$ exists by constructing it explicitly. If we want to give $P_{2}$ in such a way to maximize the capability to translate points from $L_{3}$ and thus produce a 


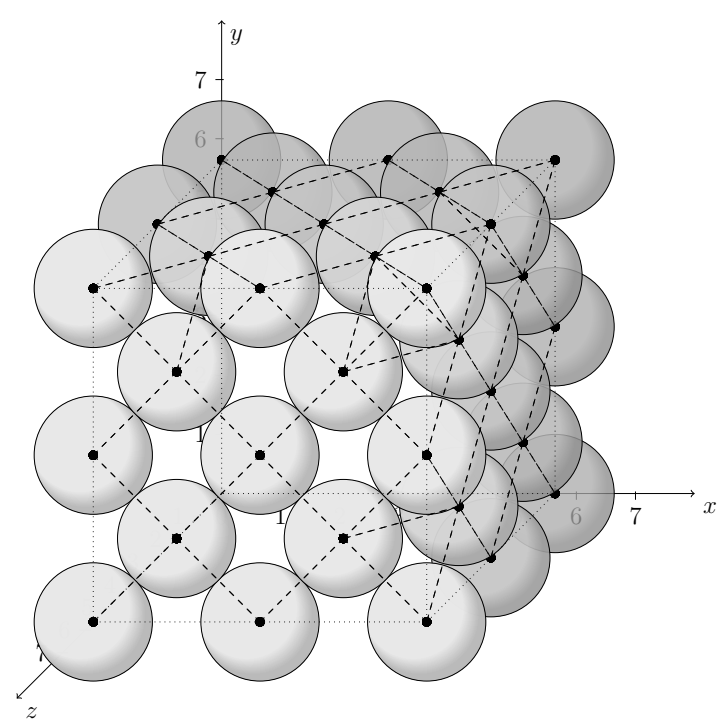

(a) $L_{5}$

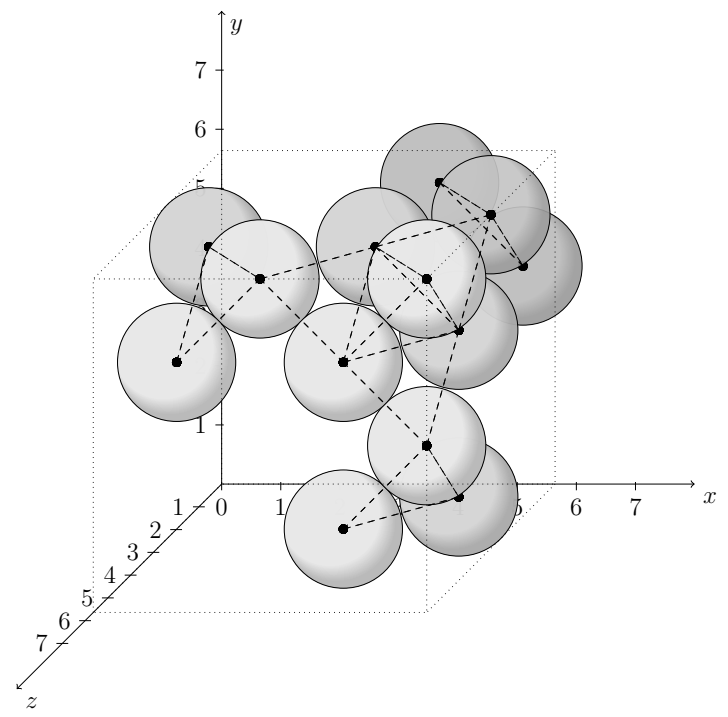

(c) $L_{5,2}$

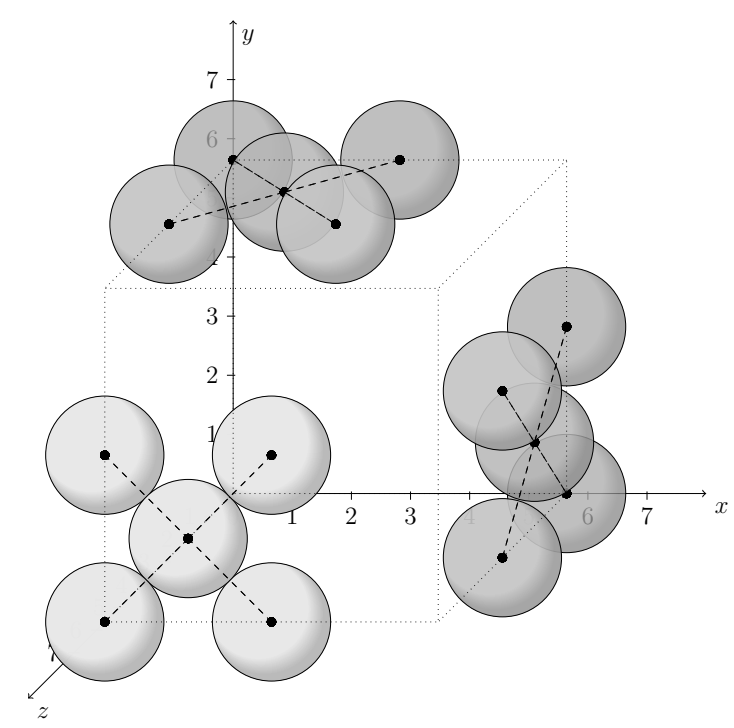

(b) $L_{5,1}$

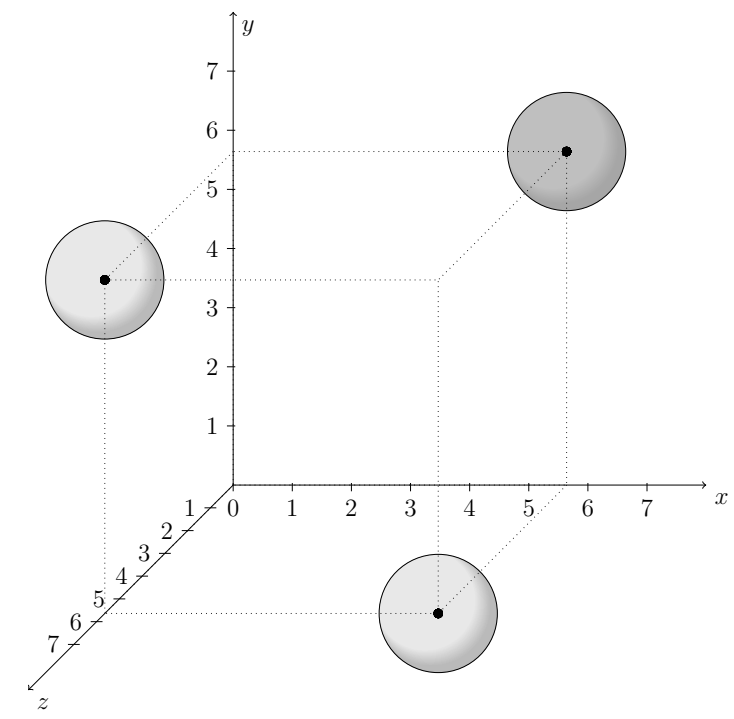

(d) $L_{5,3}$

Figure 2: An illustration of arrangements $L_{5}, L_{5,1}, L_{5,2}$ and $L_{5,3}$.

better packing $P_{3}$, we have to find the positive root of the polynomial

$$
a^{4}+4 a^{3}+8 a^{2}-8=0, a>0 \Longrightarrow a=0.818425 \ldots
$$

then $P_{2}$ is given as $P_{2}=\{\langle 0,0, a\rangle,\langle b, b, 0\rangle\}$, where $b=\sqrt{2-a^{2} / 2}$.

While $P_{p-1}$ and $L_{p}$ are separated (2), we can try to translate all points from $L_{p}$ and 
keep (3), and (4) true. We split $L_{p}$ into three subsets, and perform the translations on each of them while maintaining the given conditions.

Let $L_{p}=L_{p, 1} \cup L_{p, 2} \cup L_{p, 3}$ such that

$$
\begin{gathered}
L_{p, 1}=\left\{\left\langle l_{1}, l_{2}, l_{3}\right\rangle \in L_{p}: l_{i}, l_{j} \leqslant(p-3) \sqrt{2} \text { for some distinct } i, j \in\{1,2,3\}\right\}, \\
L_{p, 2}=\left\{\left\langle l_{1}, l_{2}, l_{3}\right\rangle \in L_{p} \backslash L_{p, 1}: l_{1}, l_{2}, l_{3}>0\right\}, \\
L_{p, 3}=L_{p} \backslash L_{p, 1} \backslash L_{p, 2} .
\end{gathered}
$$

For an illustration of sets $L_{5}, L_{5,1}, L_{5,2}$ and $L_{5,3}$, see Figure 2 .

Now we can give $T_{p}$ as

$$
\begin{gathered}
T_{p}=T_{p, 1} \cup T_{p, 2} \cup T_{p, 3}, \\
T_{p, i}=\left\{\left\langle l_{1}-u_{i, 1} \tau_{i}(p), l_{2}-u_{i, 2} \tau_{i}(p), l_{3}-u_{i, 3} \tau_{i}(p)\right\rangle:\left\langle l_{1}, l_{2}, l_{3}\right\rangle \in L_{p, i}\right\}, \\
u_{1, j}=\left\{\begin{array}{ll}
1 & \text { if } l_{j}=(p-1) \sqrt{2} \\
0 & \text { otherwise }
\end{array}, u_{2, j}=1, u_{3, j}=\left\{\begin{array}{ll}
1 & \text { if } l_{j} \neq 0 \\
0 & \text { otherwise }
\end{array},\right.\right.
\end{gathered}
$$

where $\tau_{1}(p), \tau_{2}(p)$ and $\tau_{3}(p)$ are small numbers such that $\tau_{1}(p)>\tau_{2}(p)>\tau_{3}(p)$ and conditions (3) and (4) hold. If we additionally state that $h\left(P_{p-1}, T_{p, 1}\right)=0, h\left(P_{p-1} \cup\right.$ $\left.T_{p, 1}, T_{p, 2}\right)=0$ and $h\left(P_{p-1} \cup T_{p, 1} \cup T_{p, 2}, T_{p, 3}\right)=0$, we can give explicit solutions for $\tau_{1}, \tau_{2}$ and $\tau_{3}$ as follows

$$
\begin{gathered}
\tau_{1}(3)=2 \sqrt{2}-2-a, \\
\tau_{2}(p)=\frac{\sqrt{2}}{3}\left(\frac{\tau_{1}(p)}{\sqrt{2}}+2-\sqrt{-\tau_{1}(p)^{2}+2 \sqrt{2} \tau_{1}(p)+4}\right), \text { for all } p \geqslant 3, \\
\tau_{3}(p)=\frac{\sqrt{2}}{2}\left(\sqrt{2} \tau_{2}(p)+1-\sqrt{-\tau_{2}(p)^{2}+2 \sqrt{2} \tau_{2}(p)+1}\right), \text { for all } p \geqslant 3, \\
\tau_{1}(p)=\sqrt{2}+\tau_{3}(p-1)-\sqrt{-\tau_{3}(p-1)^{2}+2 \sqrt{2} \tau_{3}(p-1)+2}, \text { for all } p>3 .
\end{gathered}
$$

We notice that $D\left(P_{p}\right)=D_{p}-\tau_{3}(p)$, hence the maximum separation distance can be given as

$$
d_{n} \geqslant \frac{2}{(p-1) \sqrt{2}-\tau_{3}(p)}, \text { for all } n \leqslant g(p)-2
$$

and while $\tau_{3}(p)>0$, then $d_{n}>\sqrt{2} /(p-1)$.

We denote the lower bound of improvements by

$$
I_{p}=d_{g(p)-2}-d_{p}^{\prime} .
$$

By performing the calculations, we get particular values such as $I_{3}>8.235 \cdot 10^{-11}$, $I_{4}>1.276 \cdot 10^{-79}, \ldots$. This is a rough approximation while condition (4) needs to remain true for all $p$ and does not allow us to further improve the packing for particular $p$. In the next section, we show that $I_{p}$ is usually above this approximation.

We did not find a way to improve the packings when $n=g(p)-1$, and we conjecture that in this case $d_{n}=d_{p}^{\prime}$. 


\section{Optimization Approach}

Most of the existing packing methods focus on searching for a completely new arrangement of spheres, usually performing a search from a randomly given initial position of spheres. Such approach assumes that the packing of higher density can be reached after a certain number of iterations and multiple runs of the search procedure using different initial parameters $[2,5,7]$. The large number of iterations often limits the search procedure to the use of double or quadruple floating-point precision, to maintain computation speed. This precision is insufficient to detect improvements in many packings. Many of these approaches are adapted and modified from widely known procedures for packing congruent circles in a square or a circle $[1,4,8,9,13]$.

The method we suggest is based on a hypothesis that an improved packing can be reached just by the omission of two or more spheres from the ccp and by performing a translation of spheres using the available space made after we remove the spheres.

The initial positions of the sphere centers we denote by $S_{p, r} \subset G_{p}$, as a set of $g(p)-r$ points such that at least one sphere with a center $s_{i} \in S_{p, r}$ can be continuously translated inside a cube container without overlapping with other spheres. More precisely,

$$
\bigcup_{i=1}^{g(p)-r}\left\{q \in C_{p}: q \notin S_{p, r},\left(S_{p, r} \backslash\left\{s_{i}\right\}\right) \cup\{q\} \in \mathcal{F}\right\} \neq \varnothing .
$$

We can see that the construction of $S_{p, r}$ is possible for $r \geqslant 2$, and we experimentally determine solutions based on improvements reached for certain arrangements. To simplify the search procedure, instead of trying to figure out the best performing arrangements $S_{p, r}$ for each pair $(p, r)$, we find removal patterns $R_{r}=G_{p} \backslash S_{p, r}$ and use them to search for improvements for any $p$.

Table 1 shows patterns in a simplified notation where $R_{r}^{\prime}=\left\{\frac{\sqrt{2}}{2} s: s \in R_{r}\right\}$. We notice that only $R_{2}$ is the most likely an optimal pattern for all $p>1$.

\begin{tabular}{ll}
$r$ & $R_{r}^{\prime}$ \\
\hline 2 & $\{\langle 0,1,1\rangle,\langle 1,1,0\rangle\}$ \\
3 & $\{\langle 0,0,0\rangle,\langle 1,0,1\rangle,\langle 2,0,0\rangle\}$ \\
4 & $\{\langle 0,1,1\rangle,\langle 1,1,0\rangle,\langle 1,1,2\rangle,\langle 2,1,1\rangle\}$ \\
5 & $\{\langle 0,1,1\rangle,\langle 1,0,1\rangle,\langle 1,1,0\rangle,\langle 2,0,0\rangle,\langle 2,1,1\rangle\}$ \\
6 & $\{\langle 0,0,0\rangle,\langle 0,1,1\rangle,\langle 1,0,1\rangle,\langle 1,1,0\rangle,\langle 2,0,0\rangle,\langle 2,1,1\rangle\}$ \\
\hline
\end{tabular}

Table 1: Experimentally determined patterns $R_{r}^{\prime}$

Using the initial arrangement $S_{p, r}$ we try to perform the translation of each sphere using the limited set of translation vectors denoted by $T$. This algorithm can be described as follows: 
- For a given initial set $S_{n} \leftarrow S_{p, r}$ repeat until $D\left(S_{n}\right)<D_{p}$ :

- For each $s_{i} \in S_{n}$ do:

- Randomly choose $t \in T$,

- Let $v=\left\{s_{i}+k t: k \in[-1,1]\right\}$,

- Let $v_{i}=\left\{q \in v:\left(S_{n} \backslash\left\{s_{i}\right\}\right) \cup\{q\} \in \mathcal{F}, D\left(S_{n} \cup\{q\}\right)=D\left(S_{n}\right)\right\}$,

- Find endpoints $a$ and $b$ of the largest line segment $\overline{a b} \subseteq v_{i}$ such that $s_{i} \in \overline{a b}$,

- New position of $s_{i}$ is given as $s_{i} \leftarrow(a+b) / 2$.

After we try to move all points from $S_{n}$, we say that we completed one iteration. Because of the very limited space to which we translate the spheres, and in order to minimize the number of required iterations, we usually set $T=\{0,1\}^{3} \backslash\{\langle 0,0,0\rangle\}$. We also tested performances when $T$ takes different values such as $\{-1,0,1\}^{3}$, more or less reduced sets, but the improvements gained were always slightly worse.

In practice, $D\left(S_{p, r}\right)$ is slightly larger than $D_{p}$ while coordinates are given with finite precision. The described procedure stops when the first improvement is detected, but if we continue the search, we can improve the packing even more. It is also important to set the precision above the expected value of $D_{p}-D\left(S_{p, r}\right)$, otherwise the improvement cannot be registered. Choosing the higher precision enables us to reach an improvement with less iterations, but only up to a certain level. We usually set the precision 1.5 times higher than the expected improvement. If the precision is too high, the search can be very slow, thus often we have to guess the range of possible improvements using a lower precision at first, and increase it if an improvement cannot be reached.

This approach is different from the procedures used for sphere packing in the past, as in $[2,5,7,12]$, while we focus only on tiny changes/improvements in the high density structure. We cannot consider this approach as a good general packing method for $r \geqslant p$. Its main weakness is that, because of the small available space where spheres can be moved, random perturbations are hard to implement, or at least we did not find any good method to do it. Still, this method allows us to find improved packings in less than one second for some well examined cases even when high precision is not required, as for example $n=29,59$ or 60 .

The improvements attained are shown in the Tables 2 and 3. Table 2 lists values obtained for $I_{p}$ with $4 \leqslant p \leqslant 21$. Because of the slow computation times for $p \geqslant 13$, we ran a search with approximately 5000 iterations when improvement gains started to slow down. Table 3 lists other improved packings for $3 \leqslant r \leqslant 6, r<p$ and $4 \leqslant p \leqslant 12$ using $R_{r}$ patterns described in Table 1 . The results are listed as the best known values performed after a large number of iterations and multiple runs of the search procedure. 


\begin{tabular}{lll}
$n$ & $p$ & $I_{p}$ \\
\hline 30 & 4 & $7.34 \cdot 10^{-68}$ \\
61 & 5 & $7.18 \cdot 10^{-80}$ \\
106 & 6 & $2.26 \cdot 10^{-314}$ \\
170 & 7 & $9.09 \cdot 10^{-622}$ \\
254 & 8 & $3.74 \cdot 10^{-629}$ \\
363 & 9 & $7.51 \cdot 10^{-629}$ \\
498 & 10 & $5.00 \cdot 10^{-2584}$ \\
664 & 11 & $9.67 \cdot 10^{-2563}$ \\
862 & 12 & $1.76 \cdot 10^{-4988}$ \\
1097 & 13 & $1.70 \cdot 10^{-5020}$ \\
1370 & 14 & $2.01 \cdot 10^{-5044}$ \\
1686 & 15 & $1.29 \cdot 10^{-5076}$ \\
2046 & 16 & $1.78 \cdot 10^{-5116}$ \\
2455 & 17 & $3.30 \cdot 10^{-5047}$ \\
2914 & 18 & $1.47 \cdot 10^{-10118}$ \\
3428 & 19 & $4.20 \cdot 10^{-10121}$ \\
3998 & 20 & $1.16 \cdot 10^{-20344}$ \\
4629 & 21 & $2.46 \cdot 10^{-20582}$ \\
\hline
\end{tabular}

Table 2: Improved values of $I_{p}$

\begin{tabular}{llll|llll}
$n$ & $p$ & $r$ & $d_{n}-d_{p}^{\prime}$ & $n$ & $p$ & $r$ & $d_{n}-d_{p}^{\prime}$ \\
\hline 29 & 4 & 3 & $2.23 \cdot 10^{-12}$ & & & & \\
\hline 59 & 5 & 4 & $1.95 \cdot 10^{-11}$ & & & & \\
60 & 5 & 3 & $2.09 \cdot 10^{-20}$ & & & & \\
\hline 103 & 6 & 5 & $3.38 \cdot 10^{-14}$ & & & & \\
104 & 6 & 4 & $9.98 \cdot 10^{-47}$ & & & & \\
105 & 6 & 3 & $1.34 \cdot 10^{-76}$ & & & & \\
\hline 166 & 7 & 6 & $3.08 \cdot 10^{-21}$ & 494 & 10 & 6 & $4.77 \cdot 10^{-57}$ \\
167 & 7 & 5 & $7.72 \cdot 10^{-31}$ & 495 & 10 & 5 & $6.96 \cdot 10^{-199}$ \\
168 & 7 & 4 & $1.59 \cdot 10^{-87}$ & 496 & 10 & 4 & $1.72 \cdot 10^{-310}$ \\
169 & 7 & 3 & $8.49 \cdot 10^{-148}$ & 497 & 10 & 3 & $1.28 \cdot 10^{-605}$ \\
\hline 250 & 8 & 6 & $2.98 \cdot 10^{-28}$ & 660 & 11 & 6 & $2.83 \cdot 10^{-119}$ \\
251 & 8 & 5 & $1.99 \cdot 10^{-43}$ & 661 & 11 & 5 & $1.99 \cdot 10^{-173}$ \\
252 & 8 & 4 & $3.11 \cdot 10^{-102}$ & 662 & 11 & 4 & $1.40 \cdot 10^{-407}$ \\
253 & 8 & 3 & $6.02 \cdot 10^{-153}$ & 663 & 11 & 3 & $3.47 \cdot 10^{-615}$ \\
\hline 359 & 9 & 6 & $3.84 \cdot 10^{-28}$ & 858 & 12 & 6 & $8.40 \cdot 10^{-248}$ \\
360 & 9 & 5 & $6.45 \cdot 10^{-44}$ & 859 & 12 & 5 & $2.68 \cdot 10^{-404}$ \\
361 & 9 & 4 & $2.05 \cdot 10^{-101}$ & 860 & 12 & 4 & $7.74 \cdot 10^{-745}$ \\
362 & 8 & 3 & $1.08 \cdot 10^{-152}$ & 861 & 12 & 3 & $2.91 \cdot 10^{-1212}$ \\
\hline
\end{tabular}

Table 3: Improvements reached using patterns $R_{r}$ 


\section{References}

[1] D. Boll, J. Donovan, R. L. Graham, B. D. Lubachevsky. Improving Dense Packings of Equal Disks in a Square. Electron. J. Combin. 7: \#R46, 2000.

[2] Th. Gensane. Dense Packings of Equal Spheres in a Cube. Electron. J. Combin. 11: \#R33, 2004

[3] M. Goldberg. On the densest packing of equal spheres in a cube. Mathematics Magazine 44: 199-208, 1971

[4] A. Grosso, A. R. M. J. U. Jamali, M. Locatelli, F. Schoen. Solving the problem of packing equal and unequal circles in a circular container. J. Glob. Optim. 47: 63-81, 2010

[5] W. Huang, L. Yu. Serial Symmetrical Relocation Algorithm for the Equal Sphere Packing Problem. arXiv:1202.4149, 2012

[6] A. Joos. On the packing of fourteen congruent Spheres in a cube. Geom. Dedicata 140: $49-80,2009$

[7] M. Locatelli, M. Maischberger, F. Schoen. Differential evolution methods based on local searches. Comput. Oper. Res. 43: 169-180, 2014

[8] M. C. Markot, T. Csendes. A New Verified Optimization Technique for the "Packing Circles in a Unit Square" Problems. SIAM J. Optim. 16: 193-219, 2005

[9] M. C. Markot, T. Csendes. A Reliable Area Reduction Technique for Solving Circle Packing Problems. Computing 77: 147-162, 2006

[10] J. Schaer. On the densest packing of spheres in a cube. Canad. Math. Bull. 9: 265-270, 271-274, 275-280, 1966

[11] J. Schaer. On the densest packing of ten congruent spheres in a cube. In: Intuitive Geometry (Szeged, 1991), Colloq. Math. Soc. Janos Bolyai 63: 403-424, 1994

[12] E. Specht. Packings of equal spheres in fixed-sized containers with maximum packing density. http://packomania.com, 2013

[13] P. G. Szabo, M. C. Markot, T. Csendes, E. Specht, L. G. Casado, I. Garcia. New Approaches to Circle Packing in a Square. Springer, 2007 